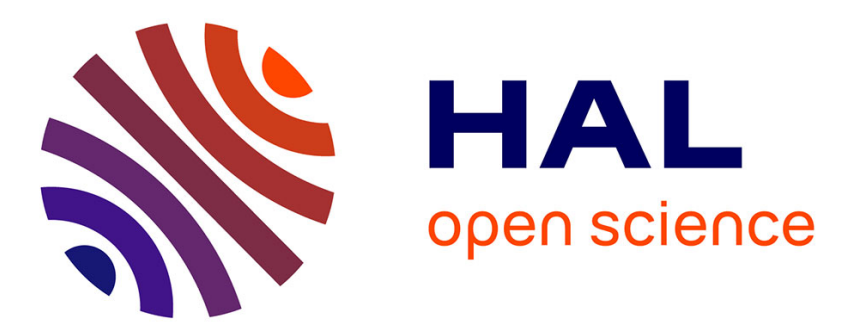

\title{
Antennas Design for the Characterization of an UHF Remotely Powered UWB Pulse Generator
}

\author{
Matthieu Egels, R. Vauche, E. Bergeret
}

\section{To cite this version:}

Matthieu Egels, R. Vauche, E. Bergeret. Antennas Design for the Characterization of an UHF Remotely Powered UWB Pulse Generator. 2019 PhotonIcs \& Electromagnetics Research Symposium - Spring (PIERS-Spring), Jun 2019, Rome, Italy. pp.1142-1148, 10.1109/PIERSSpring46901.2019.9017524 . hal-02498524

\section{HAL Id: hal-02498524 \\ https://hal.science/hal-02498524}

Submitted on 20 Dec 2021

HAL is a multi-disciplinary open access archive for the deposit and dissemination of scientific research documents, whether they are published or not. The documents may come from teaching and research institutions in France or abroad, or from public or private research centers.
L'archive ouverte pluridisciplinaire HAL, est destinée au dépôt et à la diffusion de documents scientifiques de niveau recherche, publiés ou non, émanant des établissements d'enseignement et de recherche français ou étrangers, des laboratoires publics ou privés. 


\title{
Antennas Design for the Characterization of an UHF Remotely Powered UWB Pulse Generator
}

\author{
M. Egels ${ }^{1}$, R. Vauche $^{1}$, and E. Bergeret ${ }^{1}$ \\ ${ }^{1}$ Aix-Marseille Univ, Univ Toulon, CNRS, IM2NP, France
}

\begin{abstract}
In the context of an UHF remotely powered UWB tag, this paper presents the design of the UHF antenna used to harvest energy on the tag side, and the design of the antenna used to receive emitted UWB pulses on the reader side. They have been used to characterize an UHF remotely powered UWB pulse generator whose the measurement results conclude this article.
\end{abstract}

\section{INTRODUCTION}

With the grow of the internet of things and its billions of smart devices, battery less radio links become an attractive concept. In this context, the combination of the Ultra-WideBand (UWB) impulse radio and the UHF Radio Frequency IDentification (RFID) can become a major competitor for short range communications. Indeed, it has been demonstrated with help of simulations that the power consumption of an UWB pulse generator can be low enough to be remotely powered, and thus advantages of the both wireless technologies to be associated [1]. However, due to the high power levels used in UHF-RFID, it is difficult for a remote reader to efficiently detect low energy UWB pulses without a specific UWB antenna which filters enough UHF frequencies. Moreover, cohabitation of an UWB antenna and an UHF antenna on a PCB small enough to be used as a tag is a challenge.

Based on the UWB pulse generator presented in [2] and the UWB antenna presented in [3], the system presented in [1] has been implemented, and measured in this paper. It focuses on the design of the UHF antenna used to harvest energy, and the design of the antenna used to receive emitted UWB pulses. This paper is organized as follows. In section 2, the system and its PCB is presented. Next, the design of the UHF antenna used to harvest energy required by the pulse generator is presented in section 3 and the antenna used on the reader side to receive emitted UWB pulses while filtering UHF frequencies is presented in section 4. Finally, section 5 presents the measurement results of the UHF remotely powered UWB pulse generator obtained with the designed antennas.

\section{SYSTEME ARCHITECTURE AND PCB DESIGN}

\subsection{Systeme architecture}

As shown in Fig. 1 (a), the system is an UHF-UWB transmitter tag which consists in an UHF harvesting unit and an UWB transmitter. Both have been implemented in the same chip using a $130 \mathrm{~nm}$ CMOS technology from STMicroelectronics. A top view of the designed UHF-UWB transmitter tag prototype can be seen in Fig. 1 (b).

The UWB transmitter has been designed to generate 3.1-10.6 GHz pulses and implements the filter excitation technique which allows output dynamic voltage to be higher than the voltage supply while keeping low the power consumption [4]. Its power consumption $P_{U W B}$ can be written as follows:

$$
P_{U W B}=P_{D C}+E_{A C} \cdot P R F_{\text {mean }}
$$

where $P_{D C}$ is the power consumed when no pulse is emitted, $E_{A C}$ is the additional energy consumed when a pulse is emitted, and $P R F_{\text {mean }}$ is the mean Pulse Repetition Frequency (PRF) of the emitted pulses. To supply the UWB transmitter with an UHF rectifier, the main issue comes from $P_{D C}$ which has to be reduced as far as possible to optimize the efficiency of the system. Thanks to the filter generation technique whose the used architecture is described on Fig. 2 (a), $P_{D C}$ is here only due to leakage currents since the baseband pulse generator uses only CMOS logic gates. Moreover, to reduce leakage currents, the UWB transmitter takes advantage of the used $130 \mathrm{~nm}$ technology 
by using a multi-Vt architecture [5][6] based on the High Speed (HS) and Low Leakage (LL) MOS transistors provided by the technology. For a supply voltage of $1.2 \mathrm{~V}$ and a load impedance of $50 \Omega$, this leads to a measured $P_{D C}$ of $3.91 \mu \mathrm{W}$, a measured $E_{A C}$ of $68 \mathrm{pJ}$ per pulse (which corresponds to $34 \mathrm{pJ}$ per bit for an OOK modulation), a measured peak to peak output voltage of $2 \mathrm{~V}$, and an emitted energy of $2.34 \mathrm{pJ}$ per pulse [2].

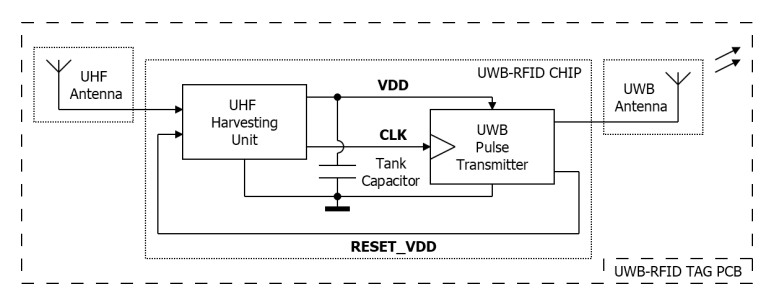

(a)

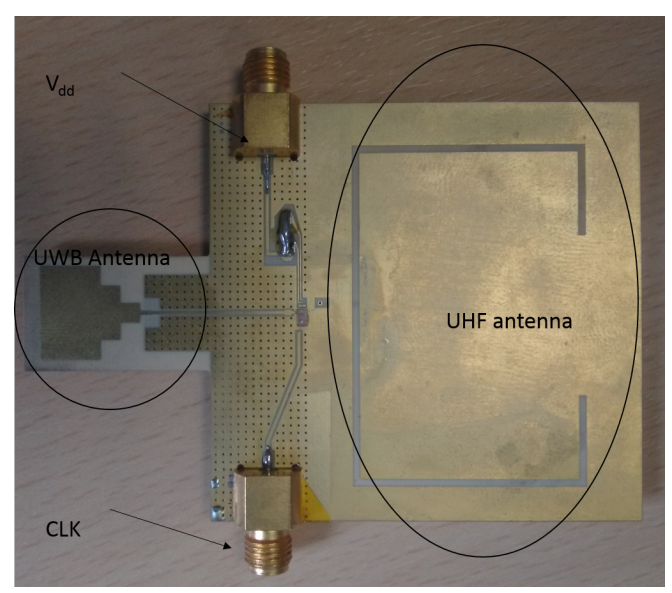

(b)

Figure 1: Bloc diagram (a) and top view photography (b) of the UHF-UWB tag.

As shown in Fig. 2 (b), the UHF harvesting unit is built around a rectifier which is based on a three stages multiplier. It has been interconnected with the UWB pulse transmitter to work as indicated in Fig. 3. Firstly, $V D D$ increases when an UHF carrier is sensed by the rectifier. Next, a rising edge occurs on $C L K$ when $V D D$ is higher than a threshold voltage fixed by the threshold detector circuit. This $C L K$ rising edge triggers the UWB transmitter wake-up and the generation of one UWB pulse. One nanosecond later, the UWB pulse generator goes into a deep sleep mode and a rising edge occurs on $R E S E T_{-} V D D$ which turns $V D D$ to $0 \mathrm{~V}$. Finally, a falling edge occurs on CLK followed by another one on RESET_VDD which allows $V D D$ to increase again. Thus, this UHF-UWB tag continuously emits UWB pulses with a PRF which is directly proportional to the power available on the UHF antenna output.

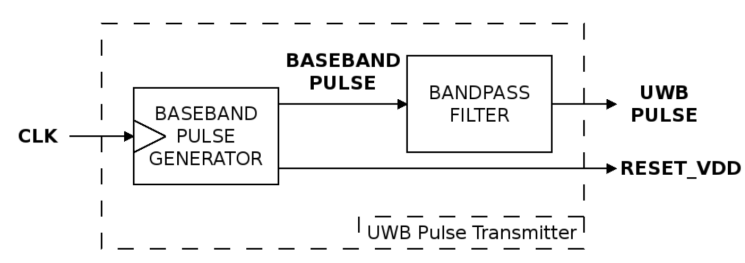

(a)

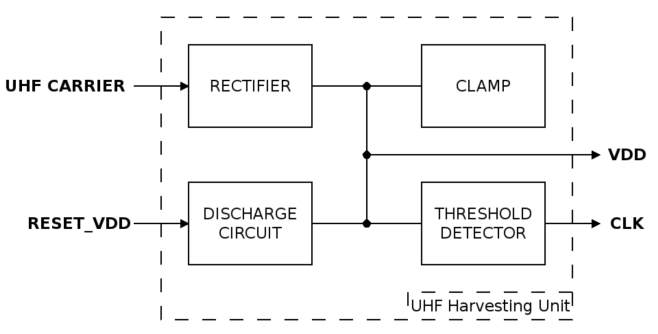

(b)

Figure 2: Bloc diagram of the UWB pulse generator (a) and bloc diagram of the UHF harvesting unit (b).

\subsection{PCB design}

The chip described previously is connected with the help of bondwires to the two required antennas. The first one is used to emit UWB pulses through the air and the second one is used to receive power from an UHF carrier for supplying the tag. These two antennas are placed on the same PCB. In order to limit losses in the UWB frequency band, a low loss Roger 4003 substrate has been chosen $\left(\epsilon_{r}=3.3\right.$ and $\left.\tan \delta=2_{-}, 10^{-3}\right)$. As the UWB antenna operates in the 3.1-10.6 $\mathrm{GHz}$ frequency band, the inlayer has been chosen in order to have mono modal Grounded Coplanar Wave Guide (G-CPW) and rigid board (see Tab. 1). The G-CPW is designed using the top first gold layers (layer 1 and 3). Layer 3 is also dedicated to the G-CPW ground and the layer 1 to the signal and lateral grounds of the G-CPW. Widths and gaps of signal lines are set to have a $50 \Omega$ characteristic impedance and vias are added to connect lateral grounds to the internal ground in 


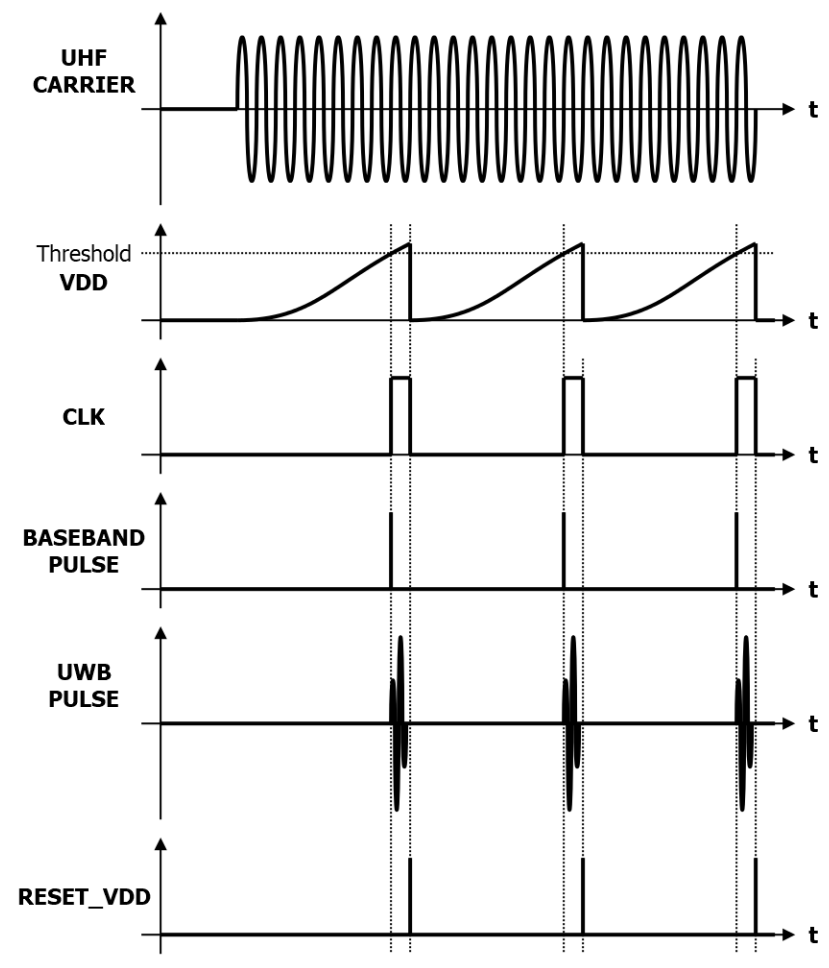

Figure 3: Main signals of the UHF-UWB transmitter tag

order to cancel spurious propagated odd mode. The design and the measurements of the 3.1-10.6 $\mathrm{GHz}$ UWB antenna are not described in this paper but are fully presented in [3] and [7] and the next section deals with the design of the UHF antenna.

Table 1: Inlay of the UHF-UWB tag

\begin{tabular}{|c||c|c|c|}
\hline \hline Layer n & material & use & thickness \\
\hline \hline 1 & copper & UWB + RFID antennas & $30 \mu \mathrm{m}$ \\
\hline 2 & Roger 4003 & dielectric & $200 \mu \mathrm{m}$ \\
\hline 3 & copper & UWB ground and RFID antenna feed & $30 \mu \mathrm{m}$ \\
\hline 4 & Roger 4003 & dielectric & $500 \mu \mathrm{m}$ \\
\hline 5 & copper & UWB ground & $30 \mu \mathrm{m}$ \\
\hline 6 & Roger 4003 & dielectric & $200 \mu \mathrm{m}$ \\
\hline 7 & copper & UWB ground & $30 \mu \mathrm{m}$ \\
\hline
\end{tabular}

\section{UHF ANTENNA DESIGN AND UHF RECTIFIER CHARACTERIZATION}

\subsection{UHF antenna design}

The UHF tag antenna has been designed to supply the tag from an UHF carrier. It has also been directly matched to the input UHF rectifier in order to cancel the losses added by a matching circuit [8]. To achieve it in a limited area (i.e. a credit card $87 \times 50 \mathrm{~mm}^{2}$ ), a slot folded dipole [8] feeded by a buried micro strip line is chosen. This topology provides a maximum peak gain in the zero azimuth direction and a quasi isotropic gain in the zero azimuth plan (see Fig. 4).

The length of the antenna is set to have a maximum radiated gain to maximize the wireless power transfer range and an inductive input impedance to match with the rectifier IC input. The final match is achieved with the optimization of the length and the width of the open ended buried microstrip line. Figures 4 (a) and (b) show respectively the top view photography of the UHF antenna prototype and its simulated radiation pattern in elevation and azimuth planes (which 


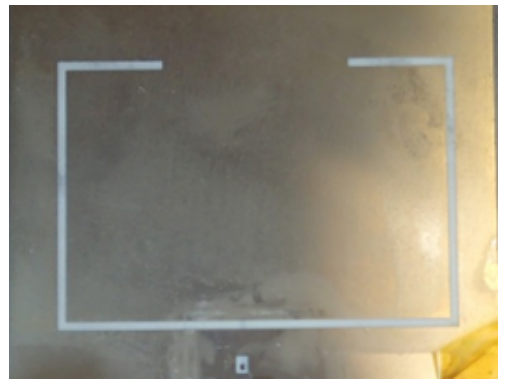

(a)

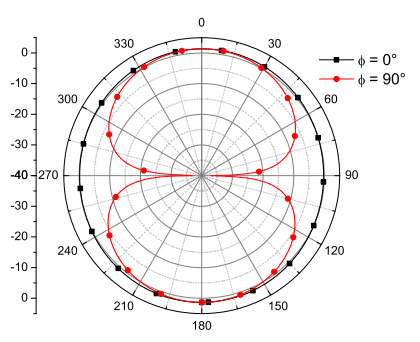

(b)

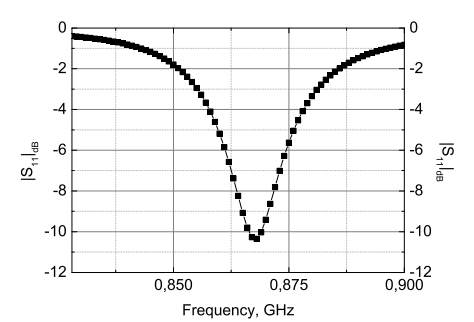

(c)

Figure 4: Top view photography (a), simulated radiation patern at $868 \mathrm{MHz}$ (b), and matching (c) of the UHF harvesting antenna.

can not be directly measured for a non $50 \Omega$ antenna). Finally, figure 4 (c) shows the simulated reflection coefficient between the antenna and the input UHF rectifier computed as follows :

$$
S_{11}=\frac{Z_{\text {ant }}-Z_{\text {ship }}^{*}}{Z_{\text {ant }}+Z_{\text {ship }}}
$$

\subsection{UHF rectifier characterization}

A manufactured prototype has been fully measured in the anechoic chamber of the lab. The measurements have been performed with a vector signal generator connected to a $40 \mathrm{~dB}$ power amplifier, itself connected to a $7 \mathrm{dBi}$ realized gain antenna through a $10 \mathrm{~m}$ long low loss cable. Finally, the rectifier has been placed at $2.5 \mathrm{~m}$ of the phase center of the test antenna. With this setup, the incident power on the rectifier antenna can be computed from the Friss formula as usually. However, only the output rectifier voltage can be measured here to characterize the performances of the UHF rectifier and the UHF antenna. Indeed, the efficiency and the output power of the rectifier cannot be computed since the current consummed by the UWB pulse generator cannot be measured. The output voltage has also been measured for several incident power $\left(P_{i n}\right)$ in the $780-880 \mathrm{MHz}$ frequency band.

As shown in Fig. 5 (a), the maximum output rectifier voltage is obtained at $815 \mathrm{MHz}$ which represents a shift down of $53 \mathrm{MHz}$ compared to simulations (i.e. $6 \%$ ). This can be explained by the deviation of the input transistor behavior from the large signal model used to design the rectifier, in addition to the length of the bondwires used to connect the IC to the antenna. An other reason can be the frequency shift between the maximum efficiency of the UHF rectifier and the minimum matching of the antenna. In addition, a voltage step can be observed in the output voltage between 0.15 and $0.6 \mathrm{~V}$. It is due to the leakage current of the pulse generator which has a peak value for this voltage range since leakage current versus supply voltage is not a monotonic function. This effect can be seen in Fig. 5 (b) too since it shows the output voltage of the rectifier as a function of the incident power at $815 \mathrm{MHz}$. Finally, the last performed measurement is the output voltage for several orientations of the UHF antenna. They have been performed in the $\mathrm{E}$ and $\mathrm{V}$ polarization planes. The results plotted on Fig 5 (c) confirm the simulated shape of the radiation pattern.

\section{READER ANTENNA}

As the maximum gain direction is the same for the UWB antenna and the UHF antenna which have been implemented on the PCB tag, a specific UWB antenna which rejects the UHF frequency band and operates in 3.1-10.6GHz frequency band has been designed.

\subsection{Design}

The main goal of this antenna is also to receive the 3.1-10.6 GHz UWB pulses emited by the pulse transmitter and to reject the UHF frequencies. To achieve it, an Archimedes spiral complimentary antenna is chosen. This antenna pattern has tree major advantages that can be exploited in the particular context of the targeted application. Firstly, it has a frequency independent behavior for the frequency band where its greater diameter is greater than $\lambda / \pi$, which means that its gain and its impedance are constant for these frequencies. Next, the radiated electromagnetic field is circular 


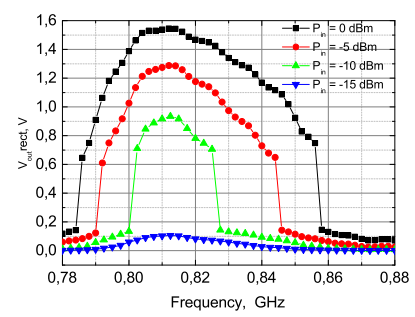

(a)

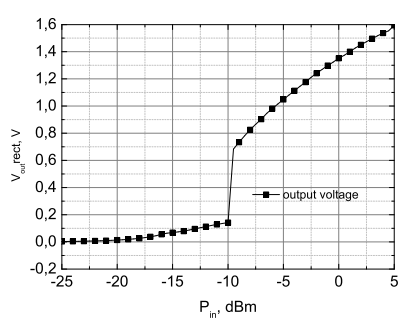

(b)

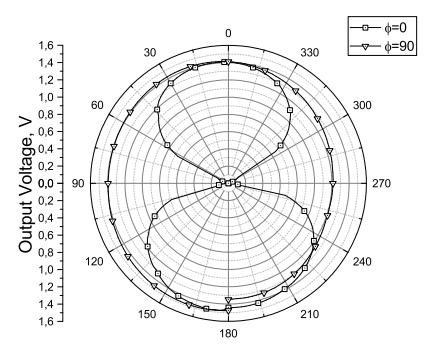

(c)

Figure 5: Output rectifier voltage as a function of the frequency for varius incident power (a), output rectifier voltage at $815 \mathrm{MHz}$ as a function of the power at $815 \mathrm{MHz}$ (b), output rectifier voltage in azimutal and elevation planes at $815 \mathrm{MHz}$ and $0 \mathrm{dBm}$ incident power (c).

for this frequency band and linear for lower frequencies. Finally, the antenna is totally mismatched for lower frequencies.

By taking into account these considerations, a spiral antenna with a flat gain and an axial ratio lower than $3 \mathrm{~dB}$ in the $3.1-10.6 \mathrm{GHz}$ frequency band can be obtained using the following steps:

1. Its diameter is set to satisfy the condition $D_{\max }>\lambda_{3.1 \mathrm{GHz}} / \pi$.

2. As the spiral must be truncated in its center for manufacturing reasons, its minimal diameter is set to satisfy $D_{\min }<\lambda_{11 G H z} / \pi$.

3. Width and gap between spiral harms are set to obtain complementary spiral antennas. This provides a constant gain and a constant input impedance of $120 \Omega$ in the $3.1-10.6 \mathrm{GHz}$ frequency band.

4. A ring is added and connected to the antenna in order to miniaturize and make constant the axial ratio and the radiated gain for the lower frequencies [9].

5. A balun is added in order to match the antenna to $50 \Omega$ in the UWB frequency band [10][11].

6. As a final step, its length is set to maximize the mismatch antenna in the UHF frequency band.

A prototype of this antenna has been fabricated on a low loss substrate (Taconic TLY with $\epsilon_{r}=2.2$ and $\tan \delta=1.910^{-4}$ ) with a laser engraving process. The Fig. 6 shows the top view of the antenna prototype and the values of the geometric parameters obtained with the design method previously described.
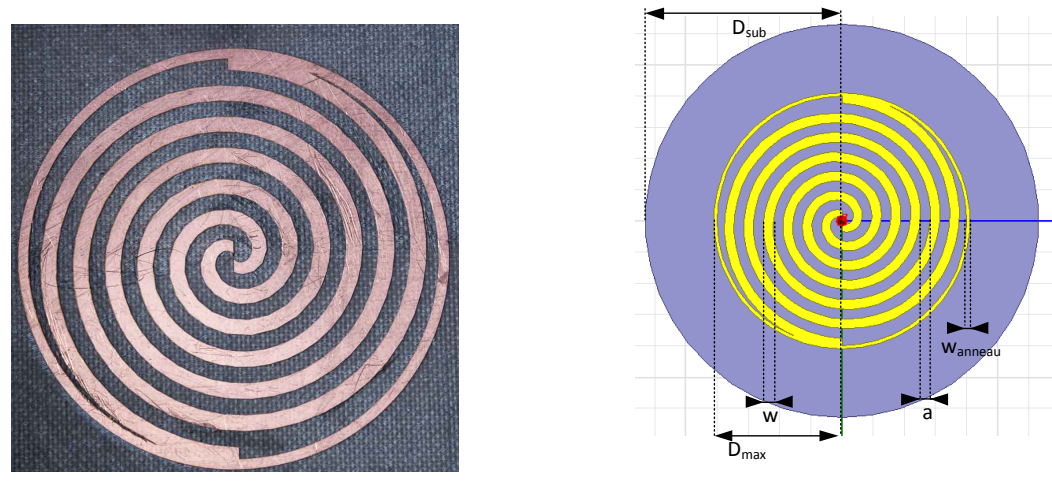

\begin{tabular}{|c||c|}
\hline Parameter & Value \\
\hline \hline$D_{\max }$ & $20 \mathrm{~mm}$ \\
\hline$D_{\text {sub }}$ & $30 \mathrm{~mm}$ \\
\hline$w$ & $1.55 \mathrm{~mm}$ \\
\hline$a$ & $1 \mathrm{~mm}$ \\
\hline$w_{\text {anneau }}$ & $1 \mathrm{~mm}$ \\
\hline turn number & 3 \\
\hline
\end{tabular}

Figure 6: Top view photography and geometrical parameters description. 


\subsection{Measurements}

The designed spiral antenna has been measured in the anechoic chamber of the lab. All measurements have been performed in the $0.5-12 \mathrm{GHz}$ frequency band using a $24 \mathrm{GHz}$ Rhode \& Schwarz vector network analyzer and a $18 \mathrm{GHz}$ wideband test antenna. The matching is plotted on Fig. 7 (a). The comparison between measured and simulated results shows a good accuracy up to 8 GHz. However, a mismatch at frequencies higher than $10 \mathrm{GHz}$ is observed but does not affect the antenna performances. As expected, the antenna is fully mismatched for the sub-GHz frequency band.

The circular realized gain and Axial Ratio (AR) have been computed by measuring the linear realized gain in several polarization planes. Results plotted on Fig. 7 (b) and (c) show that the AR is increased up to $3 \mathrm{~dB}$ for frequencies higher than $8 \mathrm{GHz}$. This causes the lower circular realized gain in the upper part of the UWB frequency band. It can be due to the use of a SMA connector to connect the antenna to the VNA but does not affect the antenna performances in the UWB frequency band.

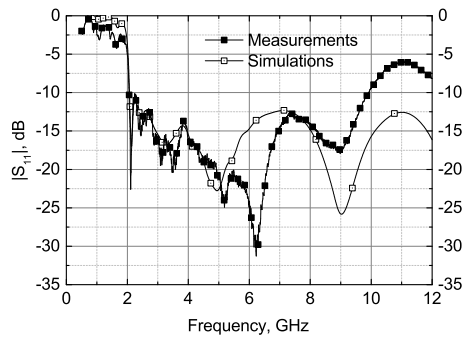

(a)

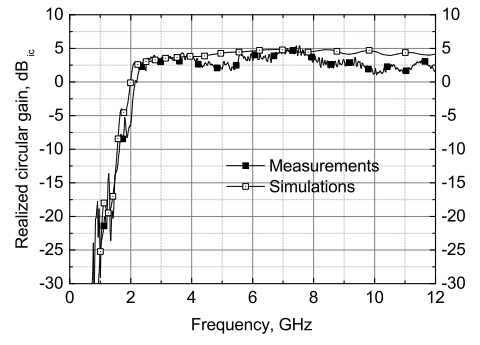

(b)

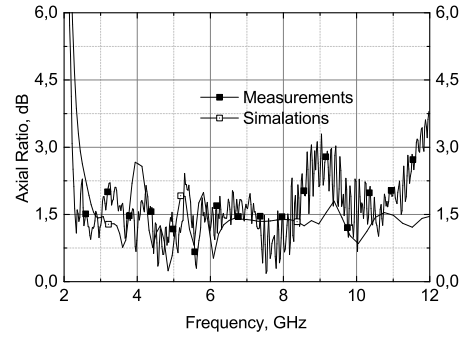

(c)

Figure 7: Simulated and measured matching of the UWB reader antenna for the $0.5-12 \mathrm{GHz}$ frequency band.

\section{UHF REMOTELY POWERED UWB PULSE GENERATOR MEASUREMENT}

In this section, the characterization of the UWB pulses emited by the UHF remotely powered UWB pulse generator is done. As a reminder, it uses as UWB antenna the one presented in [3], and as UHF antenna the one presented in section 3 of this article. In addition, it emits UWB pulses with a PRF directly proportional to the power available on the UHF antenna output since a pulse is generated when the rectifier ouput voltage is higher than a fixed threshold as shown in Fig. 3. Thus, the potential bit rate available for the UWB radio link can be measured in this configuration by the mesurement of the pulse repetition frequency.

To measure the continuously emitted pulses, an UWB receiver is emulated in the anechoic chamber by a real time $12 \mathrm{GHz}$ oscilloscope, the UWB reader antenna presented in section 4, and a 26 GHz LNA which allows UWB pulses to be highlighted from oscilloscope noise floor. The LNA gain which has been measured with the cables used to capture the UWB pulses is equal to $20 \mathrm{~dB}$ and the UWB (resp. UHF) reader antenna has been placed at $0.2 \mathrm{~m}$ (resp. $2.5 \mathrm{~m}$ ) of the UHF-UWB tag. As at $6.5 \mathrm{GHz}$ the UWB tag (resp. reader) antenna has a gain of $0 \mathrm{dBi}$ (resp. $4 \mathrm{dBi}$ ), and the waves attenuation is equal to $43 \mathrm{~dB}$ for a range of $0.2 \mathrm{~m}$, the power attenuation of UWB pulses is equal to $19 \mathrm{~dB}$. This is confirmed by the UWB pulse shown in Fig. 8 (a) which has been measured on the oscilloscope since its energy can be estimated to $0.2 \mathrm{pJ}[2]$ whereas the energy of the emitted UWB pulse can be estimated to 2.37pJ [2]. Finally, as shown in Fig. 8 (b), UWB pulses are continuously emitted up to a PRF of $165 \mathrm{kpulse} / \mathrm{s}$ for an incident power between -10dBm to $5 \mathrm{dBm}$. By considering an OOK modulation, the tag can also potentially send data to the reader with a bit rate up to $330 \mathrm{kbps}$ if an incident power of $5 \mathrm{dBm}$ is received from an UHF carrier.

\section{CONCLUSION}

This article has dealed with the design of the antennas required for the characterization of an UHF remotely powered UWB pulse generator and especially, the UHF tag antenna used to harvest 


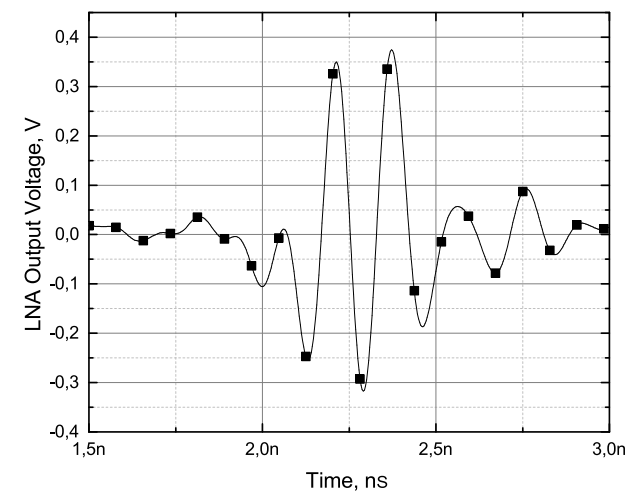

(a)

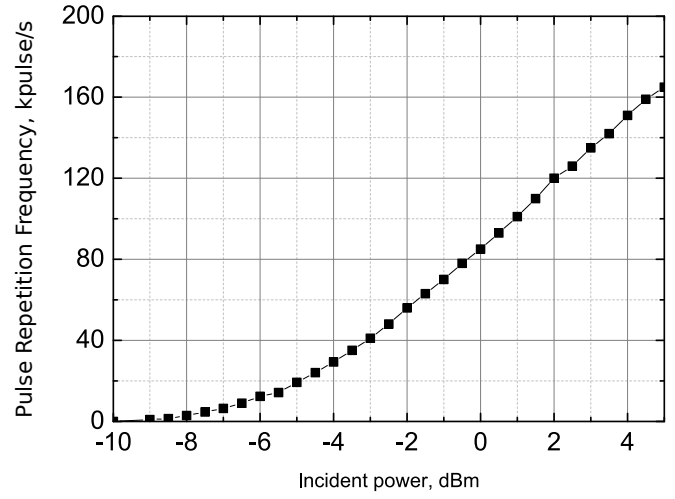

(b)

Figure 8: Example of a received pulse (a) and pulse repetition frequency of the system (b) for a distance tag-reader of $0.2 \mathrm{~m}$.

energy from an UHF carrier and the UWB reader antenna used to detect UWB pulses emitted by the tag. The main constraints were for the first one the cohabitation with the UWB tag antenna and the limited available area, and for the second one a sufficient rejection of UHF frequencies which can make difficult the detection of the emitted UWB pulses. Finally, the presented UHF-UWB tag has been characterized with the help of the both designed antennas. Measurement results show that it requires a minimum incident power of $-10 \mathrm{dBm}$ and that it can communicate with a reader at a bit rate of $330 \mathrm{kbps}$ for an incident power of $5 \mathrm{dBm}$.

\section{REFERENCES}

1. R. Vauche, E. Bergeret, J. Gaubert, S. Bourdel, O. Fourquin, and N. Dehaese, "A remotely UHF powered UWB transmitter for high precision localization of RFID tag," in UltraWideband (ICUWB), 2011 IEEE International Conference on, pp. 494-498, IEEE, 2011.

2. R. Vauche, S. Bourdel, N. Dehaese, J. Gaubert, O. R. Sparrow, E. Muhr, and H. Barthelemy, "High efficiency uwb pulse generator for ultra-low-power applications," International Journal of Microwave and Wireless Technologies, vol. 8, no. 3, pp. 495-503, 2016.

3. A. Chami, Miniaturisation et intégration d'antennes imprimées pour systèmes communicants ULB pulsés. PhD thesis, Université Nice Sophia Antipolis, 2011.

4. S. Bourdel, Y. Bachelet, J. Gaubert, R. Vauche, O. Fourquin, N. Dehaese, and H. Barthelemy, "A 9-pj/pulse 1.42-vpp ook cmos uwb pulse generator for the 3.1 2013;10.6-ghz fcc band," IEEE Transactions on Microwave Theory and Techniques, vol. 58, pp. 65-73, Jan 2010.

5. S. Mutoh, T. Douseki, Y. Matsuya, T. Aoki, S. Shigematsu, and J. Yamada, "1-v power supply high-speed digital circuit technology with multithreshold-voltage cmos," IEEE Journal of Solid-State Circuits, vol. 30, pp. 847-854, Aug 1995.

6. L. Wei, Z. Chen, K. Roy, M. C. Johnson, Y. Ye, and V. K. De, "Design and optimization of dual-threshold circuits for low-voltage low-power applications," IEEE Transactions on Very Large Scale Integration (VLSI) Systems, vol. 7, pp. 16-24, March 1999.

7. A. Chami, O. Fourquin, G. Clementi, J. Dauvignac, N. Fortino, S. Bourdel, J. Gaubert, P. Brachat, and G. Kossiavas, "Design and integration of uwb antennas for high data rate miniature impulse radio transmitter," in Antennas and Propagation (EUCAP), Proceedings of the 5th European Conference on, pp. 228-232, IEEE, 2011.

8. G. Marrocco, "The art of uhf rfid antenna design: impedance matching and size-reduction techniques," IEEE Antennas and Propagation Magazine, vol. 50, no. 1, 2008.

9. O. Ripoche, H. Aubert, A. Bellion, P. Potier, and P. Pouliguen, "Spiral antenna miniaturization in very high frequency band," in Antenna Technology and Applied Electromagnetics (ANTEM), 2012 15th International Symposium on, pp. 1-5, IEEE, 2012.

10. L. Schreider, X. Begaud, M. Soiron, B. Perpere, and C. Renard, "Broadband archimedean spiral antenna above a loaded electromagnetic band gap substrate," IET microwaves, antennas 83 propagation, vol. 1, no. 1, pp. 212-216, 2007.

11. L. Schreider, Antennes à très large bande passante et de très faible épaisseur-Application à l'intégration d'antennes dans des structures de porteurs dans la bande $100 \mathrm{MHz}-1 \mathrm{GHz}$. PhD thesis, Télécom ParisTech, 2006. 